BIOBUSINESS BRIEFS

\author{
REGULATORY WATCH
}

\title{
Impact of breakthrough therapy designation on cancer drug development
}

The breakthrough therapy designation, established in 2012 by the US Congress to expedite the development of drugs that show promising early clinical evidence of benefit over available therapies, has been granted to more than 100 drug development programmes so far. Over 30 such drugs had been approved by the US Food and Drug Administration (FDA) by the end of 2015, of which more than one-third are anticancer agents. Here, we present an analysis of the impact of the breakthrough designation on key metrics for anticancer drugs, such as review time, development time, pivotal trial phase and use of additional regulatory pathways.

We compared characteristics of novel anticancer drugs approved with and without breakthrough designation from 1 January 2013 to 31 December 2015 using the online database Drugs@FDA. In this period, the FDA approved 29 novel anticancer agents: 12 (41\%) were granted breakthrough designation during the course of their development and 17 (59\%) were not (for a list of drugs reviewed, see Supplementary information S1 (box)).

Drugs with breakthrough designation were typically approved well ahead of their Prescription Drug User Fee Act (PDUFA) goal dates (median 2.9 months before) compared with those without the designation (median 0.2 months), a difference of nearly 3 months (FIG. 1a).

Pre-market development time, calculated as the number of years from submission of an investigational new drug application (IND) to submission of a new drug application (NDA) or biologics license application (BLA), was considerably shorter among approved breakthrough-designated drugs (median 5.2 years) than non-designated drugs (median 7.4 years), a difference of 2.2 years (FIG. 1 b).

Of the 12 approved oncology drugs with breakthrough designation, 8 (66\%) were approved based on Phase I or Phase II data. By contrast, 4 of 17 (24\%) of drugs without breakthrough designation were approved on the basis of Phase II data, and none on the basis of Phase I data (FIG. 1c).

All of the drugs with breakthrough designation received priority review (100\%, 12 of 12), compared with nearly three-quarters of drugs without the designation $(71 \%, 12$ of 17). Use of the accelerated approval pathway was more varied, with three-quarters of breakthrough-designated drugs approved via accelerated approval (75\%, 9 of 12) compared with less than one-quarter of non-designated drugs (24\%, 4 of 17). Orphan designation was very common among both groups (FIG. 1 d).

In summary, among novel anticancer agents approved by the FDA between 2013 and 2015, we found that drugs with breakthrough designation reached the market more quickly than those without the designation owing to faster pre-market development and review times. We also found that considerably more breakthrough-designated drugs were approved via the accelerated approval pathway than non-designated drugs, and that breakthrough-designated drugs were more often approved on the basis of Phase I or Phase II trials. Thus, we conclude that the breakthrough designation is helping to speed patient access to innovative new cancer treatments. We also conclude that, owing to the large number of accelerated approvals among breakthrough-designated drugs,
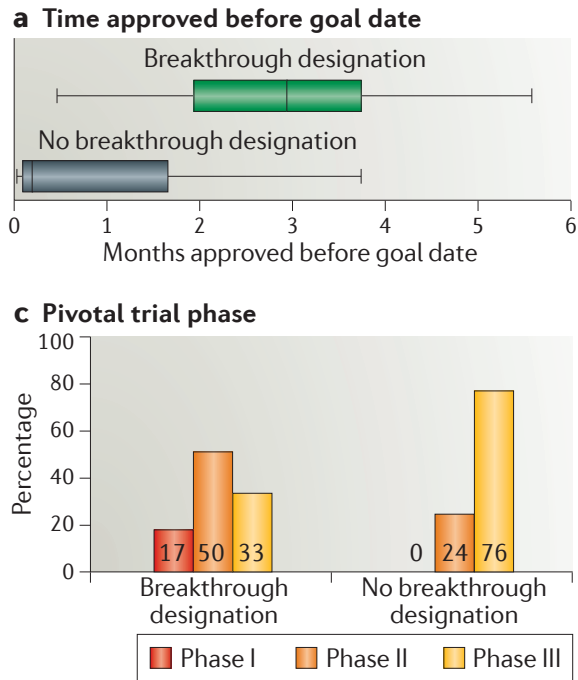

the FDA is more willing to take measured risks in approving drugs that show early evidence of substantial improvement over available therapy.

Owing to the large proportion of breakthrough-designated drugs that received accelerated approval, it can only be stated that drugs that have received a breakthrough designation have had a shorter median pre-market clinical development time, not total development time. This is because development is not over at the time of approval for drugs approved via the accelerated approval pathway, for which the FDA requires post-market confirmatory trials. In addition, the data set is small, necessitating caution in drawing conclusions on the extent to which breakthrough designation decreases pre-market clinical development time. Nevertheless, the data presented here provide preliminary evidence of the positive impact of the breakthrough therapy designation in oncology.

Michael Shea, Linda Ostermann, Ryan Hohman, Samantha Roberts, Marina Kozak, Ryan Dull, Jeff Allen and Ellen Sigal are at Friends of Cancer Research, 1800 M St NW, Suite 1050 South, Washington DC 20036, USA Correspondence to M.S. mshea@focr.org

doi:10.1038/nrd.2016.19 Published online 2 Mar 2016

The authors declare no competing interests.

SUPPLEMENTARY INFORMATION

See online article: $\underline{\mathrm{S} 1}$ (box)

ALL LINKS ARE ACTIVE IN THE ONLINE PDF
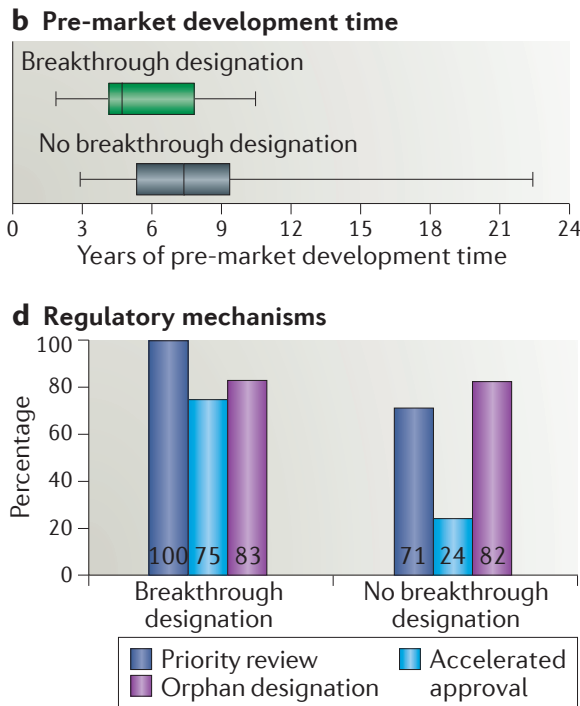

Figure 1 | Characteristics of novel anticancer agents with and without breakthrough therapy designation, approved between 2013 and 2015. a | Speed of regulatory review, which is shown by the time between marketing approval and the Prescription Drug User Fee Act (PDUFA) goal date. $\mathbf{b} \mid$ Pre-market development times, which have been calculated as the time between first clinical studies and submission of application for marketing approval. Box plots in panels $\mathbf{a}$ and $\mathbf{b}$ show interquartile ranges in the shaded areas and maximum and minimum values in whiskers. c| Development phase of pivotal registration trial (or trials). $\mathbf{d}$ |Use of additional regulatory mechanisms for drugs with and without breakthrough therapy designation. 